\title{
Construcción de termohigrómetro prototipo con base datalogger en placa Arduino para interior de edificaciones
}

\author{
Construction of prototype thermo-hygrometer with data logger base \\ on Arduino board for interior of buildings \\ Recibido: junio 24 de 2017 | Revisado: octubre 12 de 2017 | Aceptado: noviembre 18 de 2017
}

\author{
Grover Marín Mamani ${ }^{1}$ \\ Russel A. Lozada VILCA ${ }^{\mathrm{I}}$ \\ Carlos A. Huamán Carreon ${ }^{2}$ \\ Ramiro A. Bolaños Calderón ${ }^{2}$
}

\section{RESUMEN}

Se construyó un termohigrómetro prototipo con base datalogger en placa Arduino para medir la temperatura y humedad al interior de edificaciones. Este prototipo consta de un microprocesador Arduino Mega 2560 de 256K memoria, $16 \mathrm{MHZ}$ de frecuencia, sensor DHT 22 con precisión: $\pm 0.5^{\circ} \mathrm{C}$ y $\pm 1{ }^{\circ} \mathrm{C}$ y 10 s de respuesta para temperatura, así como $\pm 2 \%$ de humedad relativa de precisión con $5 \mathrm{~s}$ de respuesta para humedad relativa. Finalmente, se adicionó un módulo lector $\mathrm{SD}$ con 32GB de memoria para el registro datalogger. Se registró la temperatura y humedad cada 10 minutos durante 24 horas y observó diferencias, estadísticamente, significativas $(p>0,05)$ para la humedad. No obstante, a pesar de las diferencias no esperadas de la variable humedad en la que se requiere seguir procedimientos metodológicos para su optimización, es posible gracias a la construcción de este termohigrómetro prototipo registrar valores similares para la temperatura de los diseños validados en el ámbito comercial. Nuestro prototipo obtenido tiene un menor costo mostrándose viable para realizar estudios sobre medición de la temperatura interior en construcciones.

Palabras clave: termohigrómetro, datalogger, DHT 22, Arduino mega 2560

\section{Abstract}

A prototype thermohygrometer was built with data loggerbaseonanArduino board to measure temperature and humidity inside buildings, which consisted of an Arduino Mega 2560 256K memory microprocessor, 16MHZ frequency, DHT 22 sensor with precision: \pm $0.5^{\circ} \mathrm{C}, \pm 1{ }^{\circ} \mathrm{C}$ and 10 s of response for temperature, as well as $\pm 2 \%$ of relative humidity of precision with $5 \mathrm{~s}$ of response for relative humidity. Finally, an SD reader module with $32 \mathrm{~GB}$ of memory for the data logger registry was added. The temperature and humidity
Universidad Nacional de Juliaca Puno, Perú gmarin@unaj.edu.pe r.lozada@unaj.edu.pe

2 Universidad Andina Néstor Cáceres Vélasquez - Puno,Perú arq_huamancarreon@yahoo.es arq_rbc@hotmail.com 
were measured every 10 minutes for 24 hours where the functionality of the thermo-hygrometer was checked; however, statistically significant differences were obtained ( $p>0.05$ ) for humidity. However, in spite of the unexpected differences with the humidity variable which it is necessary to follow methodological procedures for its optimization, was possible to build a prototype thermo-hygrometer that registered similar values for the temperature to designs validated in the commercial thermo-hygrometer market. The built prototype thermo-hygrometer was feasible and with a lower cost, which means it can be used in studies on indoor temperature measurement in buildings.

Keywords: thermo-hygrometer, data logger, DHT 22, Arduino mega 2560

\section{Introducción}

La medición de temperatura y humedad constituyen variables fundamentales en los procesos de diseño bioclimático, donde los instrumentos de monitoreo que se usan, por lo general, son de tipo analógico, (Fisher \& Kebede, 2010; Vilar et al., 2015). A pesar del avance y desarrollo en la electrónica, existe una limitación en el mercado de instrumentos y sensores que almacena los datos; aunque sus costos son elevados, y esto es uno de los retos actuales en los cuales radica la fabricación con bajo costo de inversión sin desviar la precisión y exactitud en las mediciones (Azúa, Vázquez \& Hernández, 2017).

Dentro de la implementación de sistemas de adquisición de datos, la plataforma Arduino posibilita la disminución de los costos (Fernández et al., 2013) y en que cualquier medición con esta plataforma puede ser realizada en condiciones in situ. Además, la respuesta siempre será obtenida en tiempo real (Schmale,
Fehrenbacher, Shrivastava \& Pfefferkorn, 2016). Por tanto, la plataforma Arduino resulta adecuada para llevar a cabo la adquisición y manejo de las señales provenientes de los sensores DHT (Férnandez et al., 2013; Chase et al., 2012).

El sensor de tipo DHT 22 es preciso e ideal, con su integración con la plataforma Arduino (Jordáo et al., 2017). Tanto el Arduino como sus componentes soportan condiciones ambientales extremas frente a la humedad y temperatura $\left(>70^{\circ} \mathrm{C}\right)$. Asimismo, un software y hardware de código abierto permiten al usuario tener una interfaz a plena disposición (Papantoniou \& Kolokotsa, 2016). En procesos complejos, los controladores de las microcomputadoras Arduino reemplazan a otros sistemas complejos de alto costo como es el Building Energy Management Systems (por sus siglas en inglés: BENS). El propósito de la investigación fue construir un termohigrómetro prototipo sobre base datalogger en placa Arduino para medir temperaturas y humedad en interiores de edificaciones. 


\section{Materiales y métodos}

Se desarrolló un dispositivo basado en la plataforma Arduino Mega 2560, al que se integró a los sensores de temperatura y humedad relativa. Asimismo, fueron incorporados un módulo reloj DS1307 RTC, un lector de tarjetas micro SD Arduino (data logger) y una memoria SD de $4 \mathrm{~GB}$ para la reserva de datos. Se utilizaron una pantalla LCD serial, placa electrónica y cables conectores, además, de algunos componentes de fuente para la alimentación del sistema. Se consideró una placa micro-controlador AT mega 2560 (voltaje de operación de 5 volts), regulador de voltaje integrado (alimentación externa con fuente de 7-12 volts y consumo de corriente de $40 \mathrm{~mA}$ ), capacidad de memoria de $256 \mathrm{~K}$ para alojar el programa que se ejecutó y frecuencia de reloj de $16 \mathrm{MHZ}$. Tales condiciones de trabajo son recomendadas por Azúa et al., (2017).

Se estimó la utilización de un sensor DHT 22 que ofrece mayor precisión ( Kasaei et al., 2011; Scarpa et al., 2017). El módulo lector micro SD Arduino tiene la principal función de valer como interface entre la placa Arduino y la memoria SD. El consumo de memoria es bajo y puede almacenarse por más de un año un gran censo de datos para una minoria de $4 \mathrm{~GB}$ (Torrente, 2013; Vázquez, et al., 2014).

\section{Resultados y discusión}

En la Figura 1, se muestran las conexiones de los módulos: (a) Arduino Mega 2560, (b) sensor DHT 22, (c) lector micro SD Arduino, (d) reloj DS1307 RTC y (e) display LCD HD44780 y en la Figura 2, el prototipo de termohigrómetro con datalogger.

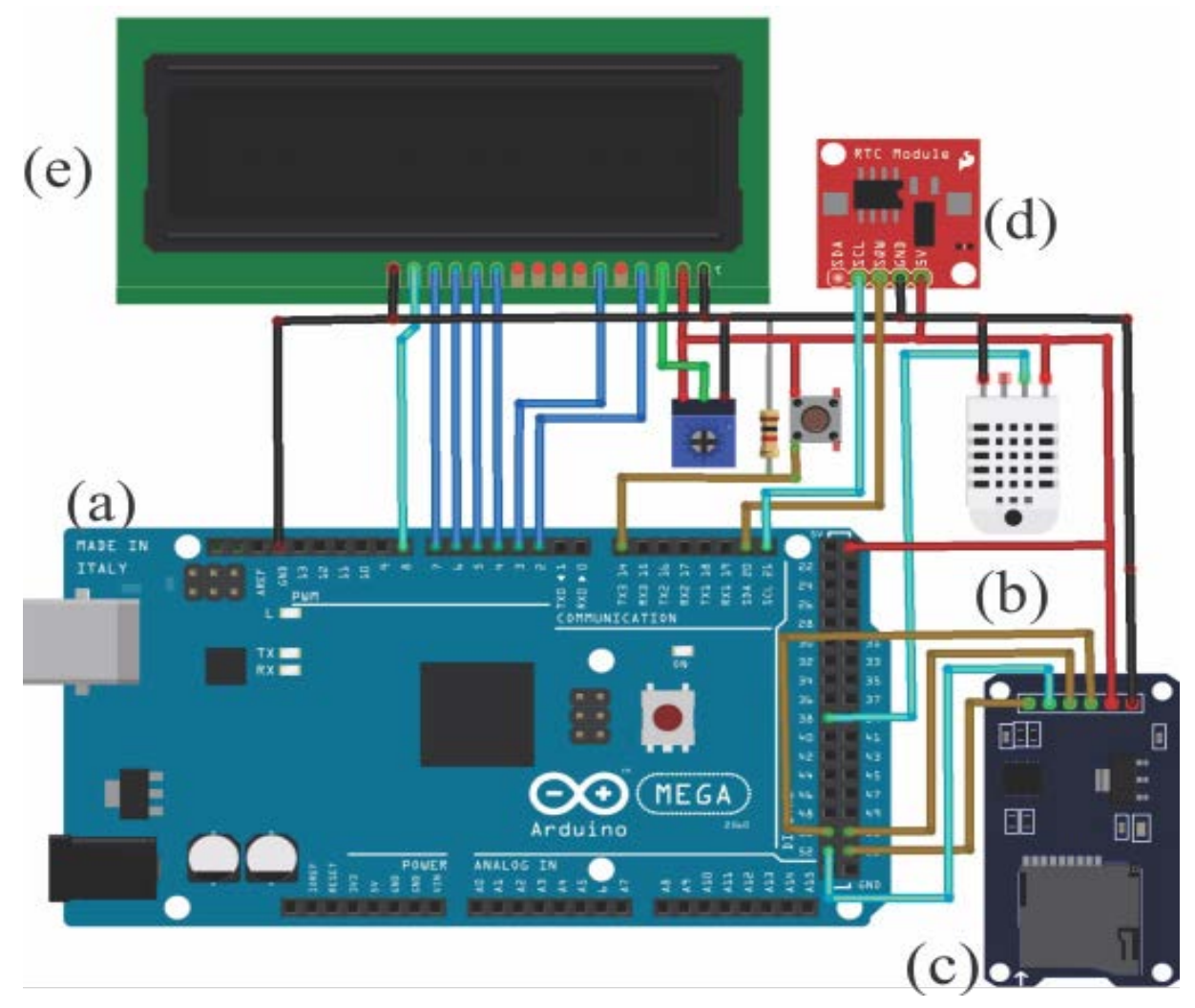

Figura 1. Prototipo de termohigrómetro 


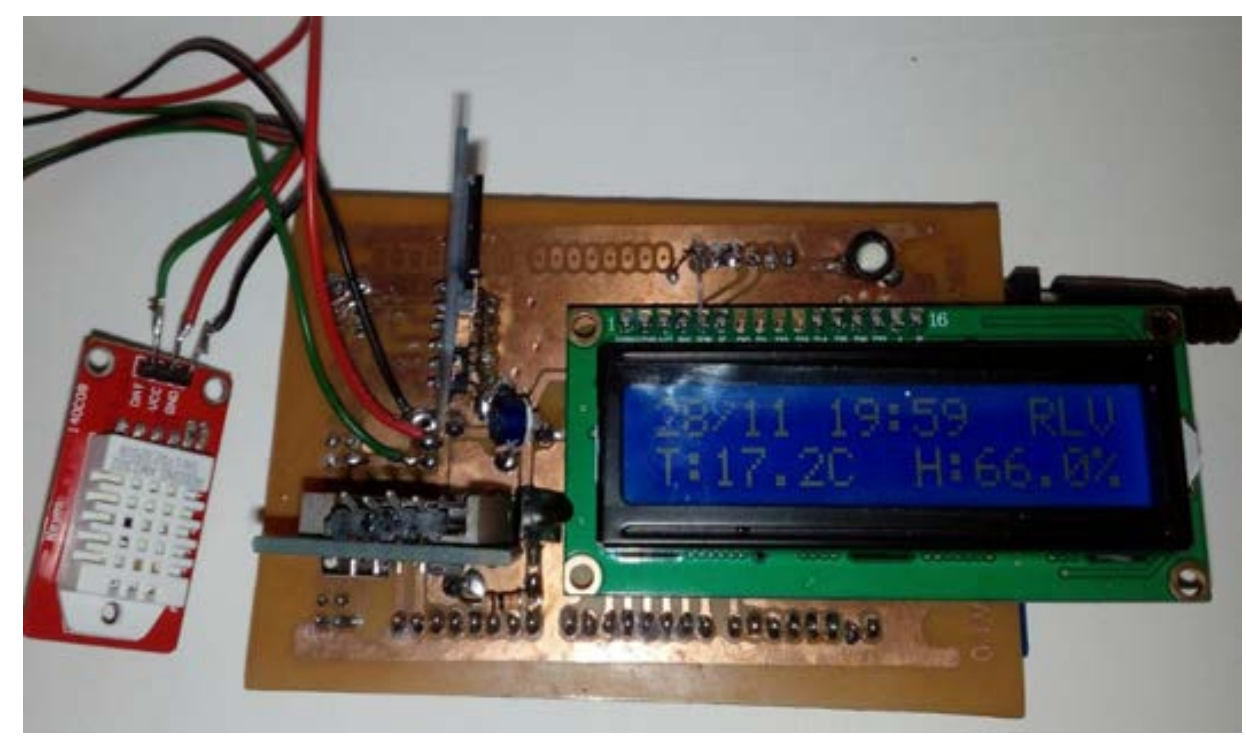

Figura 2. Prototipo termohigrómetro con datalogger

Se tomaron datos de temperatura y humedad durante 24 horas con intervalos de 10 minutos, los que fueron almacenados en una tarjeta micro SD de Arduino en formato txt.

La Tabla 1 muestra los valores registrados tanto de control y de experimento para la temperatura y humedad del termohigrómetro. El valor experimental se registró en la estación de monitoreo, estacionario, automático $472 \mathrm{CF} 72 \mathrm{C}$, ubicada en la ciudad de Juliaca, Puno que tiene como georreferencia: $15^{\circ} 28^{\prime} 15,8^{\prime \prime}$ de latitud sur y $70^{\circ} 10^{\prime} 16,4^{\prime \prime}$ longitud este y altura de 3826 m.s.n.m.

Tabla 1

Valores de temperatura y humedad (grupo control y experimental)

\begin{tabular}{rcc}
\hline Parámetro & control $^{*}$ & experimental \\
\hline Temperatura $\left({ }^{\circ} \mathrm{C}\right)$ & 8.63 & 8.70 \\
Humedad $(\%)$ & 79.58 & 93.49 \\
\hline
\end{tabular}

Fuente. Datos certificados por el Servicio Nacional de Meteorología e Hidrología del Perú: SENAMI 
La Tabla 2 muestra el resumen estadís-

tico para la temperatura y la humedad.

Tabla 2

Resumen estadistico / temperatura y humedad obtenido del termohigrómetro

\section{Temperatura}

\begin{tabular}{lcc}
\hline Estadígrafos & Experimental & Control \\
Promedio & 8.63 & 8.7 \\
Desviación estándar & 0.01 & 0.1 \\
Coeficiente de variación & $0.12 \%$ & $1.15 \%$ \\
Mínimo & 8.62 & 8.6 \\
Máximo & 8.64 & 8.8 \\
Rango & 0.02 & 0.2 \\
Sesgo estandarizado & 0.0 & 0.0 \\
\hline
\end{tabular}

Humedad

\begin{tabular}{lcc}
\hline Estadígrafos & Experimental & Control \\
Promedio & 79.58 & 93.49 \\
Desviación estándar & 0.01 & 0.01 \\
Coeficiente de variación & $0.01 \%$ & $0.01 \%$ \\
Mínimo & 79.57 & 93.48 \\
Máximo & 79.59 & 93.5 \\
Rango & 0.02 & 0.02 \\
Sesgo estandarizado & 0.0 & $-4.52194 \mathrm{E}-12$ \\
\hline
\end{tabular}

El valor del sesgo estandarizado se encontró dentro del rango esperado en los datos, lo que corresponde a una distribución normal, tal como se demuestra a continuación:
- Intervalos de confianza del 95\% intervalo de confianza para la diferencia de medias

- Suponiendo varianzas iguales: 0,07 +/$0,16 \quad[-0,09 ; 0,23]$

\section{- Comparación de medias / tempera- tura}

- Intervalos de confianza del 95\% para la media de temperatura experimental: $8,7+/-0,3[8,5 ; 8,9]$

- Intervalos de confianza del 95\% para la media de temperatura control: 8,63 $+/-0,02[8,6 ; 8,7]$

\section{- Prueba t para comparar medias / temperatura}

- Hipótesis nula: media1 $=$ media2

- Hipótesis Alt.: media1 <> media2

- Suponiendo varianzas iguales: $\mathrm{t}=1.2$ valor-P $=0.3$ 
Puesto que el intervalo contiene el valor de 0 , no hay diferencia significativa entre las medias de la temperatura control y experimental de dos muestras de datos con un nivel de confianza del $95 \%$.

- Comparación de medias / humedad

- Intervalos de confianza del 95\% para la media de temperatura experimental: $79,58+/-0,03[79,56 ; 79,61]$

- Intervalos de confianza del 95\% para la media de temperatura control: 93,49 +/- 0,03 [93,46; 93,51]

- Intervalos de confianza del 95\% intervalo de confianza para la diferencia de medias

- Suponiendo varianzas iguales: -13,91 $+/-0,02[-13,93 ;-13,89]$
- Prueba t para comparar medias I humedad

- Hipótesis nula: media1 = media2

- Hipótesis Alt.: media1 <> media2

- Suponiendo varianzas iguales: $\mathrm{t}=-1703,62$ valor- $\mathrm{P}=0,0$

Puesto que el intervalo no contiene el valor 0 , existe una diferencia estadísticamente significativa entre las medias de la humedad control y experimental con un nivel de confianza del 95\%.

La Tabla 3 muestra una comparación entre costos de termohigrómetros comerciales y el termohigrómetro prototipo construido aquí (S. / y \$ USD). La diferencia menor es de S./ 116.20 soles o $\$$ 60.54 USD y corresponde con el termohigrómetro Datalogger $\mathrm{T}^{\circ}$ y $\mathrm{H}$ Unit-t USB.

Tabla 3

Comparación entre costos de termohigrómetro comerciales y el termohigrómetro prototipo

\begin{tabular}{|c|c|c|}
\hline Prototipo & Precio S/ & $\begin{array}{l}\text { Precio } \\
\text { \$USD } \\
\end{array}$ \\
\hline Arduino Mega 2560 & 50,00 & 15,40 \\
\hline Sensor DHT 22 & 30,00 & 9,25 \\
\hline Reloj RTC DS1307 & 10,00 & 3,08 \\
\hline LCD HD44780 16*2 & 10,00 & 3,08 \\
\hline Accesorios & 20,00 & 6,17 \\
\hline Total & 130,00 & 40,12 \\
\hline \multicolumn{3}{|l|}{ Comerciales } \\
\hline Datalogger $\mathrm{T}^{\circ}$ y H Unit-t USB & 246,20 & 75,94 \\
\hline Termo higrómetro Et-175 & 424,75 & 131,00 \\
\hline Termo higrómetro Extech Rht20 & 462,35 & 142,60 \\
\hline Datalogger Cem Dt 172 & 505,35 & 155,86 \\
\hline
\end{tabular}

Se explica que los sistemas de almacenamiento de datos a través de la plata- forma Arduino pueden ser construidos a costos factibles y los dispositivos acceso- 
rios se hallan disponibles en el mercado electrónico con los que se obtienen datos fechados y ordenados en columnas en formato txt. Adicionalmente, la versatilidad y confiabilidad de la plataforma Arduino posibilita las reprogramaciones in situ, corregir errores de programación de forma rápida en periodos largos de monitoreo evitando así, se evita la pérdida de datos en las interrupciones del suministro de la red.

Finalmente, el termohigrómetro prototipo construido en este trabajo es adaptable a una batería recargable de 9 volts con panel solar (12 volts como fuente de alimentación secundaria).

\section{Conclusiones}

A pesar de la diferencia no esperada, en la variable humedad, donde se requiere seguir procedimientos para su optimización, fue posible la construcción de un termohigrómetro prototipo que registró valores similares para la temperatura en comparación con los termohigrómetros comerciales.

La construcción del termohigrómetro prototipo fue factible y a menor costo, lo que implica su empleo en estudios de medición sobre la temperatura interior en construcciones como principal finalidad.

\section{Referencias}

Azúa-Barrón, M., Vázquez-Peña, M. A., \& Hernández-Saucedo, R.A.R. (2017). Sistema de adquisición de datos de bajo costo con la plataforma arduino. Revista Mexicana de Ciencias Agricolas, 8, 1-12.

Chase, A. O., Sampaio, M. H. K., Almeida, J. F. \& Brito, de S. J. R. (2012). Data acquisition system: an approach to the amazonian environment. Latin America Transactions, IEEE, 10(2), 1616-1621.

Fernández Sánchez, S., Osorio Hernández, E., Alvarez Sánchez, E., \& Velázquez López, R. (2013). Control de Temperatura de un Invernadero a Escala mediante Programación en Arduino. Congreso $\mathrm{Na}$ cional de Control Automático, Baja California, México.
Fisher, D. K. \& Kebede, H. 2010. A lowcost microcontroller-based system to monitor crop temperature and water status. Computers and electronics in agricultura, 74, 168-173.

Jordão, M. D. L., Paiva, K. De, Firmo, H. T., Inácio, C. T., Filho, O. C. R., \& Lima, T. D. A. (2017). Low-cost automatic station for compost temperature monitoring. Revista Brasileira de Engenharia Agricola E Ambiental, 21(11), 809-813.

Kasaei, S. H., Kasaei, S. M. \& Kasaei, S. A. (2011). Design and development a control and monitoring system for greenhouse conditions based-on multi agent system. Broad Res. Art. Intel. Neur. (BRAIN), 2(4), 28-35. 
Papantoniou, S., \& Kolokotsa, D.-D. (2016). Prediction of outdoor air temperature using neural networks: Application in 4 European cities. Energy and Buildings, 114, 72-79. doi:https://doi.org/10.1016/j.enbuild.2015.06.054

Scarpa, M., Ravagnin, R., Schibuola, L., \& Tambani, C. (2017). Development and testing of a platform aimed at pervasive monitoring of indoor environment and building energy. Energy Procedia, 126, 282-288. doi: https://doi.org/10.1016/j.egypro.2017.08.155

Schmale, J., Fehrenbacher, A., Shrivastava, A., \& Pfefferkorn, F. E. (2016). Calibration of dynamic tool-workpiece interface temperature measurement during friction stir welding. Measurement: Journal of the International Measurement Confederation, 88, 331-342. doi: https://doi.org/10.1016/j.measurement.2016.02.065
Torrente, A. O. (2013). Arduino. Curso práctico de formación. Madrid, España: Alfaomega.

Vázquez, R., Robledo, A., Toledo, P., Mason, L., Mariguetti, J. \& Canali, L. (2014). Desarrollo de un procedimiento para construir un datalogger de bajo presupuesto utilizando un dispositivo genérico. II Jornada de Investigación en Ingeniería del NEA y países limítrofes, Argentina.

Vilar, A. B., de Jesus, V. L. B., de Matos, R. G., Marques, L. C. O., Zuim, F. A., Souza, J. M. de, \& Salgado, R. P. (2015). Medição de temperatura: $\mathrm{O}$ saber comum ignorado nas aulas experimentais. Revista Brasileira de Ensino de Física, 37(2), 2507-1-2507-5. doi: https://doi. org/10.1590/S1806-11173721770 